\title{
Developing Arabic License Plate Recognition System Using Artificial Neural Network and Canny Edge Detection
}

\section{Bydaa Ali Hussain}

\author{
Mohammed Sadoon Hathal ${ }^{*}$
}

Department of Computer Engineering, College of Engineering, University of Baghdad, Baghdad, Iraq.

*Corresponding author: bydaaahussain@gmail.com, ”mshm73@gmail.com.

*ORCID ID: https://orcid.org/0000-0003-4502-6928

Received 3/6/2019, Accepted 24/11/2019, Published 1/9/2020

\begin{abstract}
:
In recent years, there has been expanding development in the vehicular part and the number of vehicles moving on the roads in all the sections of the country. Arabic vehicle number plate identification based on image processing is a dynamic area of this work; this technique is used for security purposes such as tracking of stolen cars and access control to restricted areas. The License Plate Recognition System (LPRS) exploits a digital camera to capture vehicle plate numbers is used as input to the proposed recognition system. Basically, the proposed system consists of three phases, vehicle license plate localization, character segmentation, and character recognition, the License Plate (LP) detection is presented using canny edge detection algorithm, Connect Component Analysis (CCA) have been exploited for segmenting characters. Finally, a Multi-Layer Perceptron Artificial Neural Network (MLPANN) model is utilized to identify and detect the vehicle license plate characters, and hence the results are displayed as a text on GUI. The proposed system successfully detects LP and recognizes multi-style Arabic characters with rates of $96 \%$ and $97.872 \%$ respectively under different conditions.
\end{abstract}

Key words: Artificial Neural Network, Canny Edge, License Plate, Recognition System.

\section{Introduction:}

License Plate Recognition System (LPRS) is a significant application in the computer intelligent transportation field. It exploits digital image processing and detection techniques. The objective of this work is to recognize the characters from the License Plate (LP) placed on the front or back side of the car body, which is used to recognize and detect the vehicle and its owner, into text for access control and security demands $(1,2)$.

A Successful (LPRS) is based on the effective performance of software and hardware components together, hardware components are mainly composed of camera, computer and auxiliary apparatus. The LPRS works as follow: a camera will take a picture for the vehicle from the LP side when the vehicle is sensed, and hence, an image will be moved into the computer as the primary image. Computer software will pick up the LP image, then process vehicle image to obtain the LP text (3). While each country may have unique regular rules that are concerned with plates types, language, and design used, there are three general stages used to realize the aim, which are license plate localization, character segmentation, and character recognition (4). Localization process is the procedure of finding the section or region in an image that contains LP, to present the algorithm for license plate localization the color image must be converted to gray image then canny edge detection algorithm is presented, in gray scale image, an edge is defined as disjoint in gray level values to formula main object boundaries, then connect edge to rectangle based on morphology operation, the license plate is located by using geometrical feature for license plate. Segmentation phase is an important step to segment LP characters into a single character by using Connect Component Analysis (CCA). Finally, the recognition phase, we can obtain the plate number as a text accomplished by a Multi-Layer Perceptron Artificial Neural Network (MLPANN) on segment characters to recognize it, Artificial Neural Network (ANN) is an image processing technique that is influenced by the identical way of biological nervous system, similar to the brain system, that get more accuracy recognition rates with faster time recognition $(5,6$, 
7). The Arabic license plates for Arabic countries are found with different styles, Arabic or Arabic with Latin, the main LP composed of characters (Arabic letters and Indian numbers) or characters from both (Arabic and Latin letters) as well as (Arabic or Indian numbers) based on the country style (8).In this paper, Iraq LP has been used for the proposed system which has three styles written in Arabic and each style has different size and design (2).

\section{Related Works}

Muhammad Kamal, et al., (1), took advantage of the color contour algorithm and aspect ratio in located Bangladeshi license plate section, vertical and horizontal projection in segment characters, and feed forward neural network to recognize the characters and digits, the results showed that the accuracy of the system with rate was $85.56 \%$. Safaa S. Omran and Jumana A. Jarallah (2), locating License Plate was done based on intensity detection method and mathematical morphology operations, Optical Character Recognition (OCR) was used with correlation approach and template matching for character recognition, the system was investigated using 40 images, the results showed that the performance for the system was $86.6 \%$.

\section{Materials and Methods:}

The model of this work is to simulate the ALPRS performance which consists of three stages. In the first stage, the process of finding the object that belongs to LP and its location of an image, second stage is used to segment the characters (numbers, alphabets and word) into sub images, while the last stage is a character recognition, through which each character in LP image is recognized separately and displayed as a text on GUI(Fig. 1) illustrates the main stages of the LPRS system.

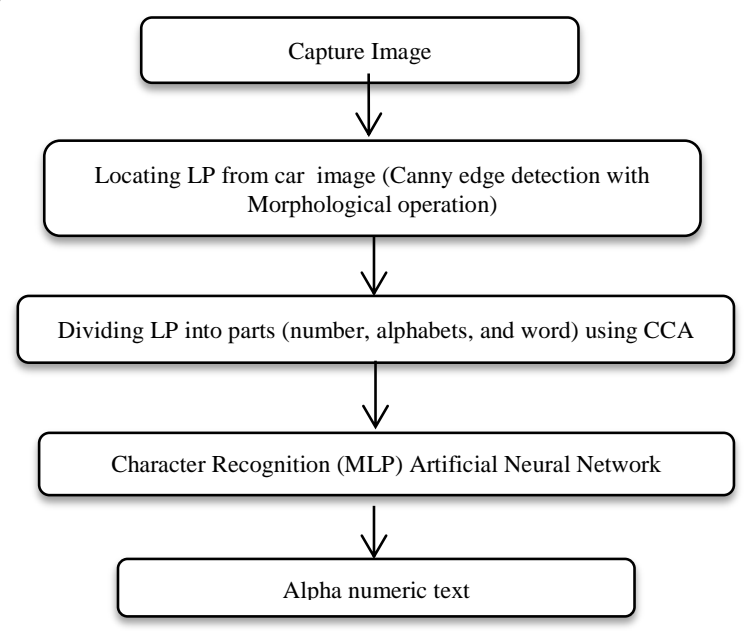

Figure 1. The main stages of LPRS model

\section{Plate Region Detection}

The main stages of detecting LP are summarized in the following steps:

- Read the image.

- Convert the image from color to gray image.

- Edge detection using the Canny method.

- Edge Finding and Linking to Rectangle.

- Candidate plate finding.

The program steps for LP detection are shown in detail in Fig. 2

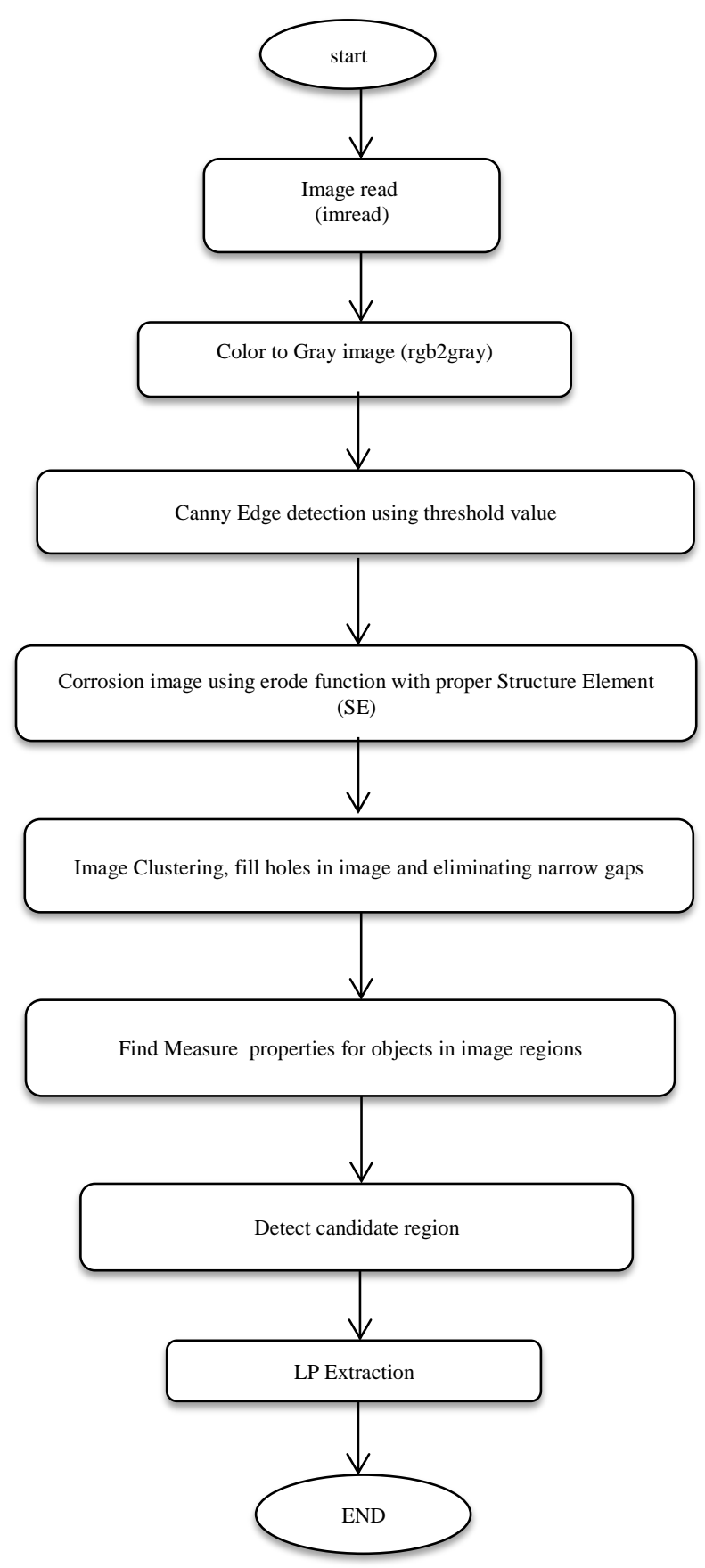

Figure 2. The flowchart of the LP algorithm

\section{a. Read Image}

Input images for License Plate Recognition System (LPRS) have been taken from Iraqi natural 
scenes and it belongs to a different type of vehicles, All the image were taken in outdoor environments and in different times of the day using day light, these pictures have been acquired using 8 megapixel iPhone 6 camera with high resolution. Fig. 3 shows some of these samples.

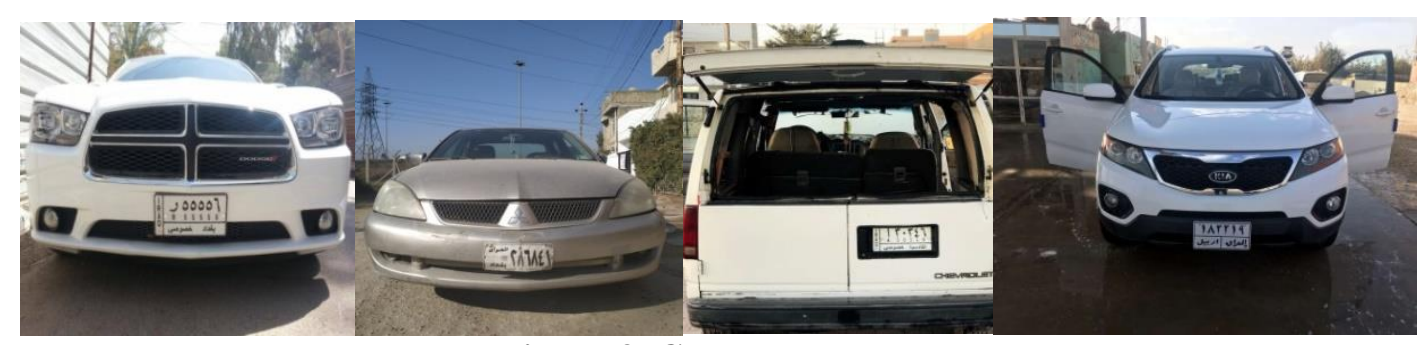

\section{b. Convert Image to Gray}

The input image to the proposed system is RGB color of JPG form which takes more time to process and largest storage space because RGB color uses 24 bit to represent each pixel, while gray image takes 8 bit to represent each pixel, so

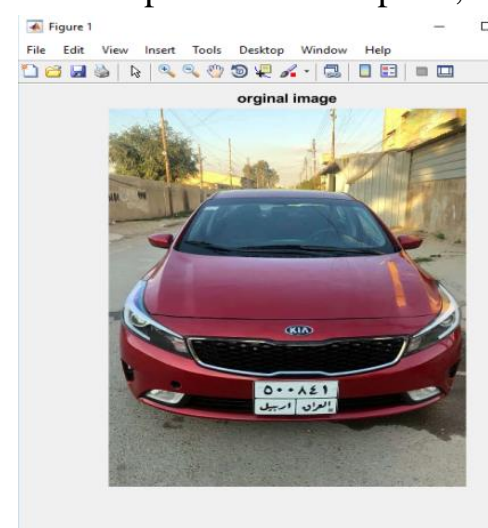

converting the image to gray image results in reducing the number of bits, where highlight on the white area found in the input image(9). Fig. 4 shows the result of a color image after transforming it to gray image.

\section{Figure (4) Gray conversion method}

\section{c. Canny Edge Detection Algorithm}

Edge is found in the image reflects a different types of information such as the border of the region, texture change, etc. With respect to LPRS, an edge detection is used to extract LP area, Canny edge algorithm is a suitable algorithm to locate a wide series of edge in a noisy image, also canny filters are applied to enhance the edge by filter useless information and preserving important information. The algorithm is used several steps $(10,11)$ :

- Filter out any noise in the original image using Gaussian filter $G_{\sigma}(m, n)$ Eq. (1)

$$
\begin{gathered}
g(m, n)=G_{\sigma}(m, n) * f(m, n) \\
\text { where } \mathrm{G}_{\sigma}(\mathrm{m}, \mathrm{n}) \text { is given by } \\
G_{\sigma}(m, n)=\frac{1}{\sqrt{2} \pi \sigma 2} \exp \left[-\frac{m 2+n 2}{2 \sigma 2}\right] .
\end{gathered}
$$

- Finding gradient intensity and its direction for the image. Eq.(2) and (3)
$\mathrm{M}(\mathrm{m}, \mathrm{n})=\sqrt{\left(g^{2}{ }_{m}(m, n)+g^{2}{ }_{n}(m, n)\right)}$

$\Theta(\mathrm{m}, \mathrm{n})=\tan ^{-1}\left[g_{n}(\mathrm{~m}, \mathrm{n}) / \mathrm{g}_{m}(\mathrm{~m}, \mathrm{n})\right]$

where $g_{m}(\mathrm{~m}, \mathrm{n})$ is first derivative in a horizontal direction and $g_{n}(\mathrm{~m}, \mathrm{n})$ is derivative in a vertical direction.

- Non-maximum suppression is applied to give a thin line in the output image and most of the noise is suppressed.

- Hysteresis, the final step in the canny algorithm is introduced to track along the other remained pixels that have not been removed and accurate in representation edge like real edges.

The details of the acquired image are controlled using the standard deviation of the Gaussian filter (Sigma), a proper threshold value (0.5) are chosen to confirm the area of LP. Fig. 5 shows canny edge detection implementation. 


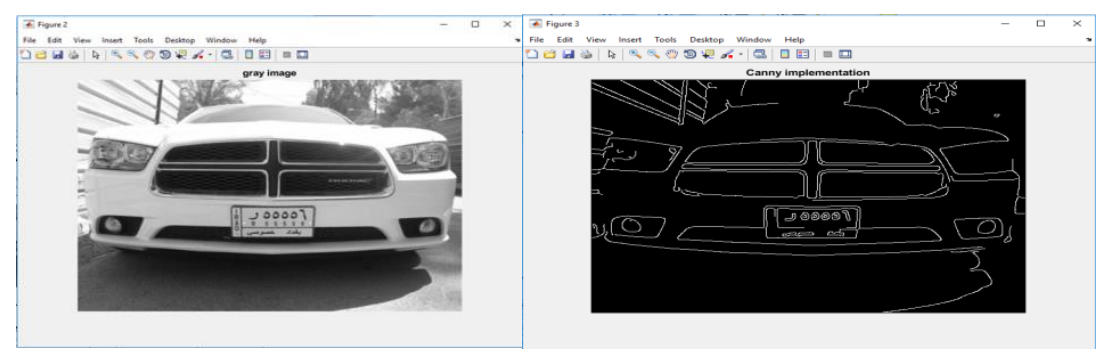

Figure (5) Canny edge implementation

\section{d. Edge Finding and Linking to Rectangle}

The main steps for finding edge and linking to rectangle are summarized as follows (12, $13)$ :

- After finding an edge for the whole image using canny filter, lines for License Plate (LP) need to be updated from the previous step, and other unrelated lines would be suppressed, corrosion behavior technique is applied in the whole image to eliminates boundary points, unrelated lines and preserve LP lines by using corrosion function with a proper linear Structure Element (SE).

- The second step is to connect LP area using closing operation with rectangle $\mathrm{SE}$, which is simply a corrosion after expansion, this operation is mainly used to obtain closed shape, eliminating small holes by filling the interior gap.
- Hence, the morphological filtered image is used to remove small objects with an area less than the LP area.

\section{e. Candidate Plate Finding}

It is the final step for localizing the LP, according to the previous step a number of candidate objects are formed with various size, it is important to find the exact location of LP object and extract it, the process is done by using the following measure properties (14):

- One of the rough positioning determines the starting position and ending position for the line and column of each object.

- To estimate the optimal object region, by comparing the region with standard LP size according to limited threshold value as shown in Table 1.

Table 1. Filtering Region Properties

\begin{tabular}{cccc}
\hline \multirow{2}{*}{ Filtering Parameters } & \multirow{2}{*}{ Candidate region (old Style LP) } & Candidate region (new Style & Candidate region (Northern LP \\
Style)
\end{tabular}

\section{Character Segmentation}

Segmentation stage is an essential part for License Plate Recognition System, this step binds each character separately preparing it for recognition process, the procedure for segment characters are describe as follow $(15,16)$ :

- Read and Resize License Plate Image.

- Binarization and Find Complement, the quality of input images is greatly different based on lighting and location conditions of the image, so there is a need to choose a tolerable threshold value in order to initiate a correct bounding box, the appropriate threshold value is chosen according to the difference between foreground and background, and hence when the significance difference appears, the selected threshold value is the best. Fig. 6a demonstrates the main step for character segmentation.

- Finding and computing the area of the bounding box, depends on Connect Component Analysis (CCA) for binary image.

- Crop out Arabic character, any box that is located within limited aspect ratio will be extracted and the others will be removed. Fig. $6 \mathrm{~b}$ shows the results for character segmentation.

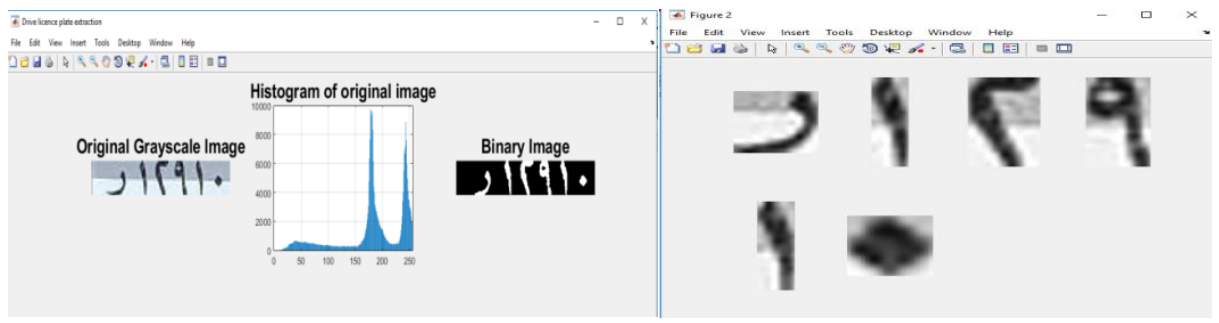

(a) (b)

Figure 6. Character segmentation 


\section{Character Recognition}

Character recognition is an essential part of the proposed system, by which the segmented characters are detected and recognized. The main algorithm of this step is Artificial Neural Networks (ANN), it contains a set of nodes from input layer connected by direct links to the output layer or to the hidden layer, and each link has a numerical weight associated with it $(17,18)$, the module of this system is a Multi-Layer Perceptron Artificial Neural Networks, the network was trained with 200 character images with different illuminations and backgrounds, all characters are used for training and testing (9x7) matrix, therefore the input to the network is 63 neurons, the 24 elements represents the network output corresponding to 14 Arabic alphabet and 10 Indian numerals, the number of nodes in a hidden layer is 100 nodes, chosen experimentally, the network has the ability to adapt with their input changing and produce best results with an iterative procedure known as Back Propagation neural network. The training system is completed when the error reached to 0.0006 , where network output fit to the desirable output.
In this work, the collected data set depends on several images with different resolution rates and sizes; due to the lack of finding a complete data set that contains Iraqi LP images, the images have been captured from 2 to 5 meters distance between camera and vehicle with angle of $90^{\circ}$ from the horizontal. The simulation tests have been done using Matlab R2018a under a development environment with Intel (R) Core i7 CPU- 4510U CPU, 2.00GHz $2.60 \mathrm{GHz}$ with $8 \mathrm{~GB}$ RAM. The experiments results have been obtained from the proposed system described previously which are mainly depending on the canny edge algorithm and MLP artificial neural network, are evaluated from test of 50 images where taken under different conditions, such as strong sunshine, shadow, cloudy weather, the vehicle, and LP have the same color, complex sense, different Iraq styles. The obtained results have been achieved with $96 \%$ as detection rate, $97.916 \%$ and $97.872 \%$ for segmentation and recognition rate respectively. Fig. 7 presents the result for the extraction plate region and recognition with different images. Giving an overall system performance of $91.99 \%$.

\section{Results and Discussion:}
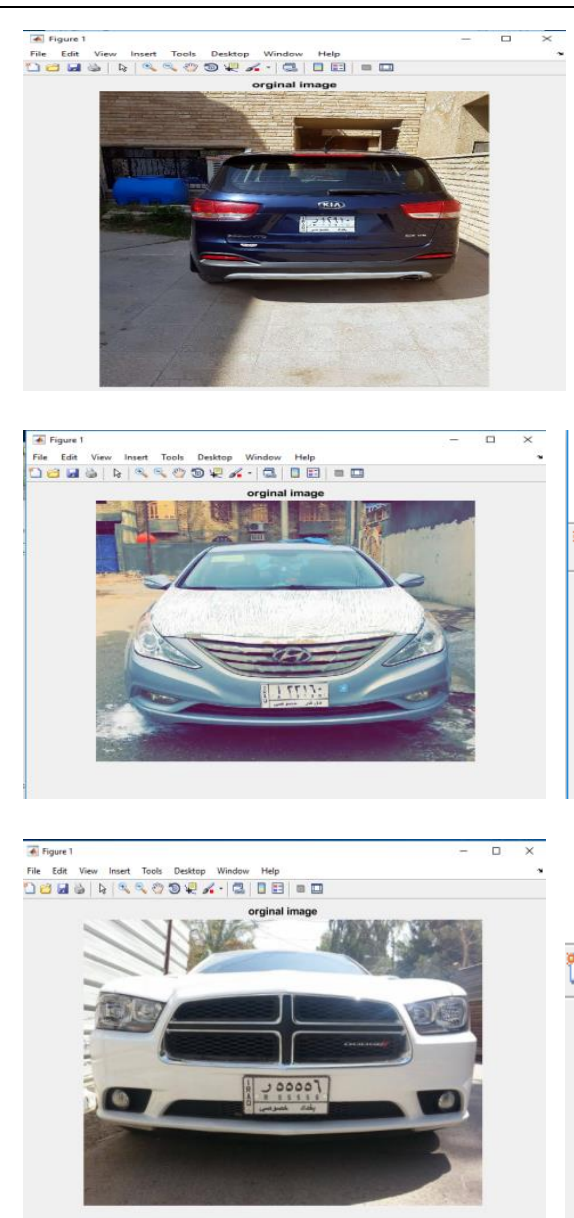
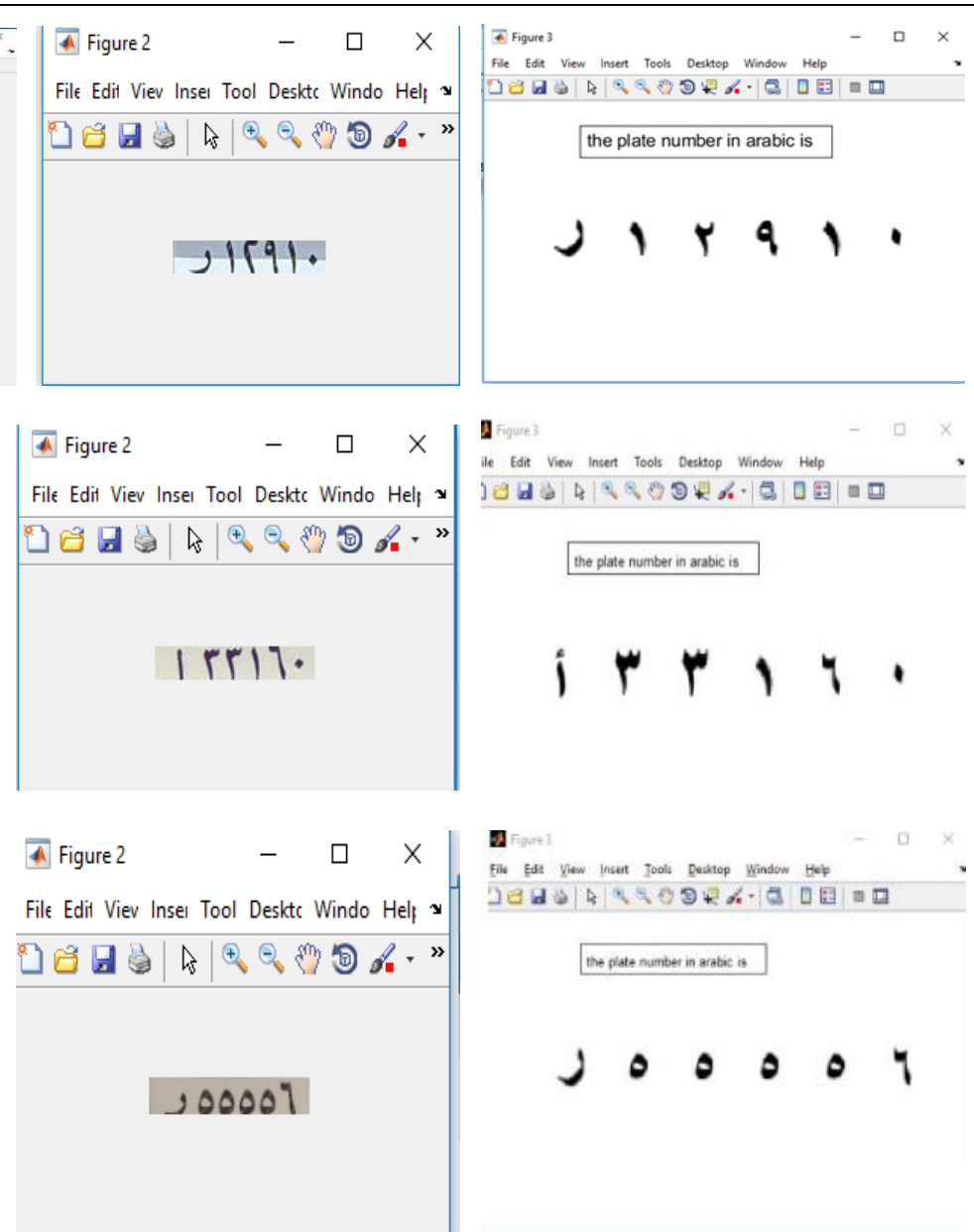

Figure 7. Experimental Result of LPRS 
Results for our developed system are compared in term of accuracy with the methods of (1) and (2) the evaluation results are shown in Table 2.

Table 2. Comparison Results

\begin{tabular}{|c|c|c|}
\hline References & $\begin{array}{l}\text { Method - P: Plate Localization, } \\
\text { R: Character Recognition }\end{array}$ & $\begin{array}{l}\text { Success } \\
\text { Rate }\end{array}$ \\
\hline (1) & $\begin{array}{l}\text { P: color contour algorithm and } \\
\text { aspect ratio. } \\
\mathrm{R} \cdot \text { feed forward neural network }\end{array}$ & $85.56 \%$ \\
\hline (2) & $\begin{array}{l}\text { P: intensity detection with } \\
\text { morphological operations. } \\
\text { R: OCR is used with correlation } \\
\text { approach and templates } \\
\text { matching. }\end{array}$ & $86.6 \%$ \\
\hline $\begin{array}{l}\text { Proposed } \\
\text { method }\end{array}$ & $\begin{array}{c}\text { P: canny edge detection with } \\
\text { morphological operations } \\
\text { R: Multi-Layer Perceptron } \\
\text { (ANN) }\end{array}$ & $91.99 \%$ \\
\hline
\end{tabular}

these results illustrate that the proposed method demonstrates better performances in comparison with the traditional algorithms and it is a promising method for future implementation.

\section{Conclusion:}

In this paper, an Arabic license plate recognition system presents different styles that belong to Iraqi license plates. In each stage, the suitable algorithms have been chosen to develop the proposed system, in this work canny edge algorithm is selected with the morphological operation that achieved better results with $96 \%$ as detection rate, this approach enable the system to zoom smoothly toward the exact frame, especially in detecting license plate for a noisy image. On the other hand MLP artificial neural network classifier is used to verify or reject the results achieving $97.872 \%$ as recognition rate; our algorithm are tested by 50 input images causing an overall system performance to be $91.99 \%$, concluded that the proposed system is outperforms the existing algorithms of license plate system.

\section{Authors' declaration:}

- Conflicts of Interest: None.

- We hereby confirm that all the Figures and Tables in the manuscript are mine ours. Besides, the Figures and images, which are not mine ours, have been given the permission for republication attached with the manuscript.

- Ethical Clearance: The project was approved by the local ethical committee in University of Baghdad.

\section{References:}

1. Hossen MK, Roy AC, Chowdhury MS, Islam MS, Deb K. License Plate Detection and Recognition system based on Morphological Approach and Feed-
Forward Neural Network. IJCSNS.2018 May 30;18(5):36-45.

2. Safaa S O, Jumana A J. Iraqi car License Plate Recognition using OCR. Annual conference on new trends in information and communications technology application. Iraq. 2017.

3. Kumar BB, Bansal M, Verma P. Designing of Licensed Number Plate Recognition system using hybrid technique from neural network \& template matching. In2015 International Conference on Computing, Communication and Security (ICCCS) 2015 Dec 4 (pp. 1-6). IEEE.

4. Khader M, SOS A, Hani S. Arabic License Plate Recognition System. Columbia International publishing, JSPTA. 2013; 1:20-42.

5. Hana M A, Marim Kh A, Amani M A, Vijay T. Saudi License Plate Recognition System using Artificial Neural Network Classifier. ICCA. Saudi Arabia. IEEE. 2017.

6. Ibrahim El K, Yousef S, Samir M O, Ali A G. An Efficient Algorithm for automatic recognition of the Lebanese car license plate. LIU. Lebanon. IEEE. 2015.

7. Bhara B, Simranjot S, Ruchi S. License Plate Recognition System using Neural Networks and Multi Thresholding Technique. IJCA. 2013 December; 84 (5).

8. Yasser $\mathrm{M}$ A. Automatic Arabic License Plate Recognition. IJCE. 2011 June; 3 (3).

9. Amir H A. An Iranian License Plate Recognition System Based on Color Features. IEEE transactions on intelligent transportation systems. 2014 August; 15 (4).

10. Pooya S H, Mojtaba S. License Plate Automatic Recognition based on Edge Detection. Faculty of computer and IT Engineering. Islamic Azad University. Qazvin branch. Qazvin. Iran. IEEE. 2016.

11. Samarth B. Implementation of vehicle License Plate recognition using canny Edge Detection. Dept. of Electronics and telecommunication. Goa College of Engineering. Ponda-Goa. India. IJCSET. 2015.

12. Mohamed Dr S, Anitha B . Accurate License Plate Localization. Department of ECE. Thiagarajar College of Engineering Madurai. ICCCET. 2011.

13. Allam M. Canny-Edge Detection Based Vehicle Plate Recognition. International journal of signal processing, Image processing, and pattern recognition. 2012 September; 5(3).

14. Abd El Rahman A, Hamdy A, Zaki F. Automatic Arabic Number Plate Recognition. second international Japan-Egypt conference on Electronics communications and computers. IEEE. 2013.

15. Erdinc K H, Kursat C K. Artificial neural networks based vehicle license plate recognition. Selcuk University. Technical Education Faculty. Konya. Turkey. Nigde Uni. Bor Vocational High School. Turkey. Elsevier Ltd. Open access under CC BY-NCND license. 2011.

16. Akoum A, Daya B, Chauvet P. Two Neural Networks for License number plates recognition. Lebanese University. Institute of technology. Lebanon. Institute 
of Applied Mathematics. France. J Theor Appl Inf Technol. 2009.

17. Richard S. Computer vision Algorithm and Applications. \#3ed. Springer. 2010 September.
18. Hamid R B. Persian Printed Numerals Classification Using Extended Moment Invariants. World Academy of Science. Engineering and Technology. IJCIE. 2010; 4(3).

\title{
تطوير نظام التعرف على لوحات السيارات العربية باستخدام الشبكة العصبية الاصطناعية و خوارزمية كاني
}

محمد سعدون حثيل

\author{
بيداء علي حسين \\ قسم هندسة الحاسبات، كلية الهندسة، جامعة بغداد، بغداد، العراق.
}

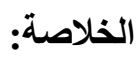

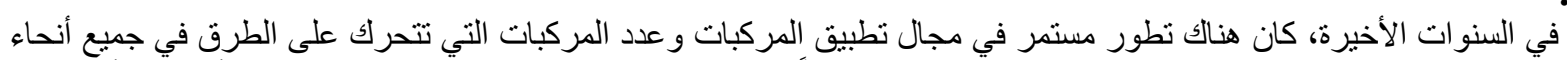

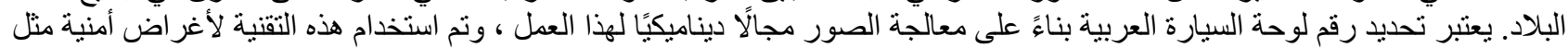

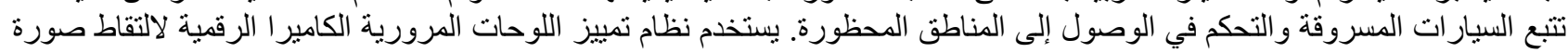

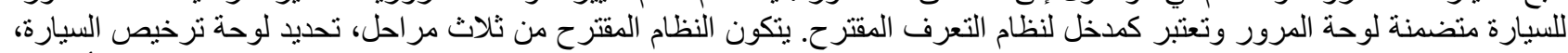

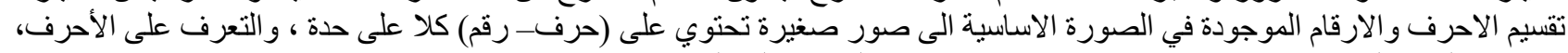

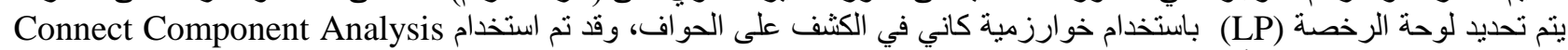

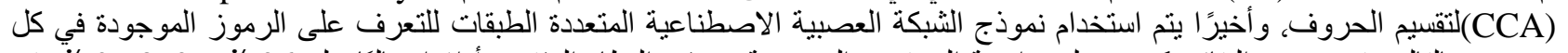

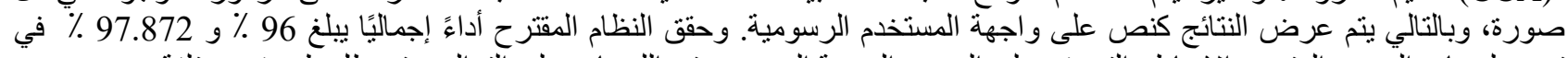

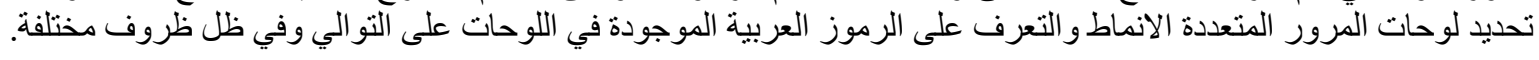
الكلمات المفتاحية: الثبكة العصبية الاصطناعية، خوارزمية كاني، لوحة الترخيص، نظام التعرف. 